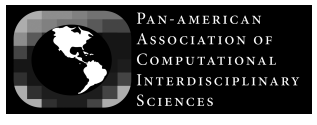

Journal of Computational Interdisciplinary Sciences (2008) 1(1):51-56

(c) 2008 Pan-American Association of Computational Interdisciplinary Sciences

ISSN 1983-8409

http://epacis.org

\title{
Combining wavelets and linear spectral mixture model for MODIS satellite sensor time-series analysis
}

\author{
Ramon M. Freitas ${ }^{1}$ and Yosio E. Shimabukuro ${ }^{2}$ \\ Manuscript received on July 26, 2008 / accepted on October 4, 2008
}

\begin{abstract}
This work presents a methodology that uses digital fraction images derived from Linear Spectral Mixture Model and wavelets transform from MODIS satellite sensor time-series for land cover change analysis. Our approach uses MODIS surface reflectance images acquired from 2000 to 2006 time period. For this study, a test site was selected in the Mato Grosso State, Brazilian Amazonia. This site has shown high deforestation rates in the last years. The samples of land cover classes were collected during four field campaigns $(2003,2004,2005$ and 2006) to be used as ground truth. The linear spectral mixture model was applied to the MODIS surface reflectance images of red surface reflectance band (620-670 nm bandwidth), near infrared surface reflectance band (NIR, 841-876 $\mathrm{nm}$ bandwidth) and medium infrared surface reflectance band (MIR, 2105-2155 $\mathrm{nm}$ bandwidth). This model generated the vegetation, shade, and soil fraction images. In the next step, the Meyer orthogonal Discrete Wavelets Transform was used for filtering the time-series of MODIS fraction images. The filtered signal was reconstructed excluding high frequencies for each pixel in the fraction images (soil, vegetation, and shade) of the time-series. This computational procedure allows to observe the original signal without clouds and other noises. The results show that wavelets transform can provide a gain in multitemporal analysis and visualization on inter-annual fraction images variability patterns.
\end{abstract}

Keywords: MODIS, Wavelets, Fractions Images, Time-Series Analysis.

1E-mail: ramon@dsr.inpe.br

2E-mail: yosio@dsr.inpe.br 


\section{INTRODUCTION}

The analysis of time series data is essential to many areas of science, engineering, finance, and economics. Wavelets analysis has been originally introduced in the fields of digital signal processing, image processing, audio recognition, and data compression as alternative to Fourier analysis. Discrete Wavelet Transform (DWT) has been used in time-series applications because it allows to characterize nonstationary process more adequately. The main advantage of the wavelets as a basis set consists on its orthonormality and localized property for signal reconstruction [8].

The Moderate Resolution Imaging Spectroradiometer (MODIS) sensor on board of NASA's Terra (EOS-AM1) and Aqua(EOS-PM1) satellites with high frequency of observations and moderate spatial resolution provides useful information for remote sensing time-series analysis. It constitutes a useful source of information for monitoring the earth surface over large areas. However, the problem of persistent cloud cover in Amazonia region breaks the ability to characterize spatial-temporal land cover changes in a fragmented forest landscape. The land cover changes have important consequences for ecosystem studies, such as carbon storage and deforestation dynamics [6].

The remote sensing time series analysis provides new vision about vegetation phenological stages. Several studies in remote sensing and signal processing demonstrated a potential of wavelets technique. The main applications concern on crop phenological studies [10], ENSO variability [7] and remove clouds algorithms [4].

A moderate or low spatial resolution data introduces the socalled "mixture problem", i.e., the pixel value is a mixture of reflectance from different targets within each pixel [11]. Several techniques, such as modeling and empirical estimations, have been applied to depict subpixel heterogeneity in land cover from remotely sensed data. In general, the Linear Spectral Mixture Model approach is a subpixel analysis and widely accepted as good indicators of vegetation properties and for landscape change detection at regional scale. This technique based on fraction images derived from different remote sensing data have provided consistent results for monitoring deforestation [12], land cover change [3], and vegetation classification [5].

The fraction images, derived from a linear spectral mixture model, constitute synthetic bands with information on endmember proportions. The generation of these images is an alternative approach to reduce the dimensionality of image data and enhancing specific information for digital interpretation $[2,11]$. The geometric accuracy of MODIS images combining with high temporal resolution provide a new approach for land cover time series studies. In this context, the objective of this paper is to present a method for land cover change analysis using the wavelets transform and linear mixture spectral model applied to the Terra MODIS daily time-series data.

\section{METHOD}

The linear spectral mixture model implements a linear relationship for the spectral reflectance mixture of surface targets within the image pixel. In this model, the pixel's values in any given spectral band is assumed to be a linear combination of the responses of the individual components in this spectral band. For any individual pixel, the linear model can be expressed by:

$$
r_{i}=\sum_{n}^{j=1} a_{i, j} x_{j}+\epsilon_{i}
$$

where $r_{i}$ represents the mean spectral reflectance in the ith spectral band, $a_{i, j}$ is the spectral reflectance of the $j$ th component in the spectral band ith (endmember), $x_{j}$ is the proportion of the jth component within the pixel and $\epsilon_{i}$ is the residual for the ith spectral band. The proportions $x_{j}$ in (1) are also subjected to two constraints:

$$
x_{j}=\sum_{n}^{j=1} x_{j}=1 \quad \text { and } \quad x_{j} \geq 0 \vee j
$$

The equation (1) can be used to form a system of $n$ linear equations, $n$ being the number of spectral bands available. Constrained least squares techniques can then be applied to estimate the components proportions $x_{j}[11]$.

The linear spectral mixture model [11] was applied on the MODIS surface reflectance images RED, NIR and MIR spectral bands for each date. A unique endmembers were used because MODIS images represents surface reflectance. The selection of end-member was based on field data and interpreter experience. This model generated soil, vegetation and shade fraction images. The soil fraction image highlights mainly non-vegetated areas (deforested clear cuts, bare soil, etc.); the vegetation fraction image shows the vegetation cover condition similar to the well known normalized difference vegetation index (NDVI); and the shade fraction image enhances water bodies, vegetation structure and burned areas.

In the next step, the Meyer orthogonal Discrete Wavelets Transform was used for filtering MODIS fraction images timeseries. The Meyer wavelet [1] (Eqs. 3-6) and scaling function (Eqs. 7-9) are defined in the frequency domain $(\omega)$. The discret form uses FIR based approximation of the Meyer Wavelet. 
Wavelet function:

$$
\begin{gathered}
\widehat{\psi}(\omega)=(2 \pi)^{-\frac{1}{2}} e^{\frac{i}{2 \omega}} \sin \left(\frac{\pi}{2} v\left(\frac{3}{2 \pi}|\omega|-1\right)\right), \\
\text { if, } \quad \frac{2 \pi}{3} \leq|\omega| \leq \frac{4 \pi}{3} \\
\widehat{\psi}(\omega)=(2 \pi)^{-\frac{1}{2}} e^{\frac{i}{2 \omega}} \cos \left(\frac{\pi}{2} v\left(\frac{3}{2 \pi}|\omega|-1\right)\right), \\
\text { if, } \quad \frac{4 \pi}{3} \leq|\omega| \leq \frac{8 \pi}{3} \\
\widehat{\psi}(\omega)=0, \quad \text { if, } \quad|\omega| \notin[0,1]
\end{gathered}
$$

where,

$$
v(a)=a^{4}\left(35-84 a+72 a^{2}-20 a^{3}\right), a \notin[0,1]
$$

Scale function:

$$
\begin{gathered}
\widehat{\phi}(\omega)=(2 \pi)^{-\frac{1}{2}}, \quad \text { if, } \quad|\omega| \leq \frac{2 \pi}{3} \\
\widehat{\phi}(\omega)=(2 \pi)^{-\frac{1}{2}} e^{\frac{i}{2 \omega}} \cos \left(\frac{\pi}{2} v\left(\frac{3}{2 \pi}|\omega|-1\right)\right), \\
\text { if, } \quad \frac{2 \pi}{3} \leq|\omega| \leq \frac{4 \pi}{3} \\
\widehat{\phi}(\omega)=0, \quad \text { if, } \quad|\omega| \geq \frac{4 \pi}{3}
\end{gathered}
$$

The filtered signal was reconstructed excluding high frequencies for each pixel in the fraction images (soil, vegetation and shade) time series. This procedure allows a viewing of original signal without clouds and other noises. The time-series of cloud-free composite images at 8-day intervals provide vegetation phenology information to identify different land cover types from the unique patterns of vegetation and soil fractions. The classes analyzed were deforested, cropland, pasture, regrowth (secondary forest) and forest. All computational procedures used the MATLAB environment.

\section{EXPERIMENTS}

\subsection{Remote Sensing Data and Processing}

The study area is located in the center northern part of the Mato Grosso State, Brazilian Amazonia (Figure 1), and comprising approximately $145,000 \mathrm{~km}^{2}$. The test site encompasses several landscape types as tropical forest, savanna, transitional forest, regrowth (segundary forest), deforested areas, croplands and pasture. This site presented high deforestation rates in the last years. The samples of deforestation land cover classes were collected during four field campaigns (2003, 2004, 2005 and 2006) to be used as ground truth. The red lines in Figure 1 represent the field samples.

The time-series of MODIS/Terra surface reflectance images are from collection 4 of 8-day L3 Global product at $250 \mathrm{~m}$ resolution (MOD09A1), acquired from 2000 to 2006. 256 images were used in this study. This MODIS product is an estimate of the surface spectral reflectance for each band, as it would have been measured at ground level with no atmospheric scattering or absorption effects, generated by applying the atmospheric correction algorithm. The MOD09 product includes the RED and NIR bands with originally $250 \mathrm{~m}$ spatial resolution and MIR bands resampled from $500 \mathrm{~m}$ to $250 \mathrm{~m}$ spatial resolution.

The monthly accumulated rainfall data, used to support the interpretation of vegetation phenological responses, were derived from Global Precipitation Climatology Project and Tropical Rainfall Measuring Mission (TRMM) [9].

The Figure 2 shows an example of signal decomposition for vegetation fraction of forest data set. The signal was decomposed on 8 scales. The high frequencies components of signal are related to noise data produced by MOD09 8-day composite algorithm or clouds contamination. The low frequencies are related to seasonal and phenological variations. In this context, the filtered signal was reconstructed excluding high frequencies (detail 1 to 4) for each pixel.

Figure 3 presents longitudinal transect on forest area. The transect shows the season and phenological variations of forest cover and deforestation process. The low vegetation value represents a deforested area. In this context, the proposed methodology showed that it was possible to identify when deforestation occurred and the pos-deforestation land use types (croplands, pasture or regrowth), i.e., to monitor the land cover change dynamics.

The vegetation fraction images time-series (Figure 4) show spatio-temporal dynamics for forest and deforested areas. The dark areas represent non vegetation areas and deforestation process, while the green represent the vegetation fraction of forest areas until the beginning of deforestation process (Figure $3 \mathrm{c}$ and d). The images can be used for land cover change spatio-temporal analysis because the noise or cloud contamination were eliminated by wavelet transform filtering.

Figure 5 shows vegetation fraction time series and monthly accumulated rainfall $(\mathrm{mm})$. This Figure presents landcover change dynamics of a deforestation process. The accumulated 


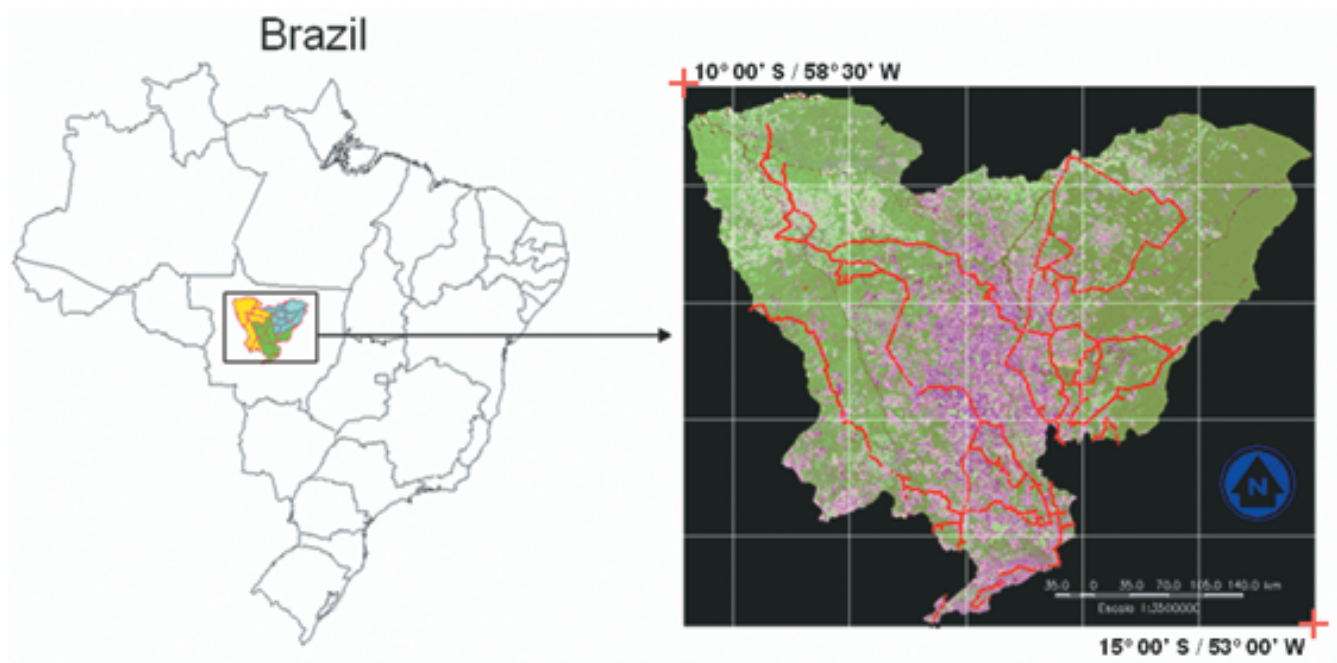

Figure 1 - Location of the study area.
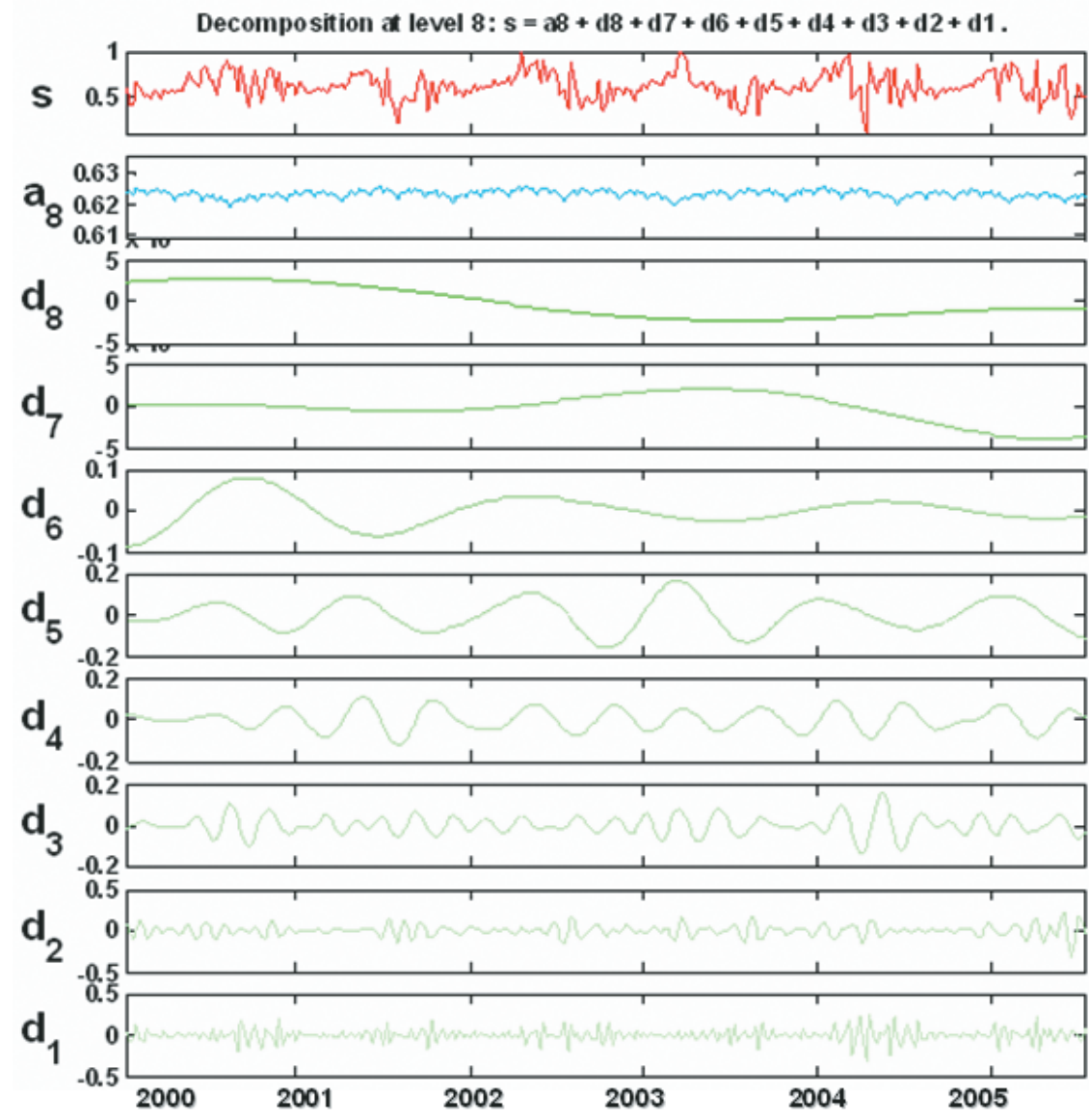

Figure 2 - Vegetation fraction signal decomposition. 


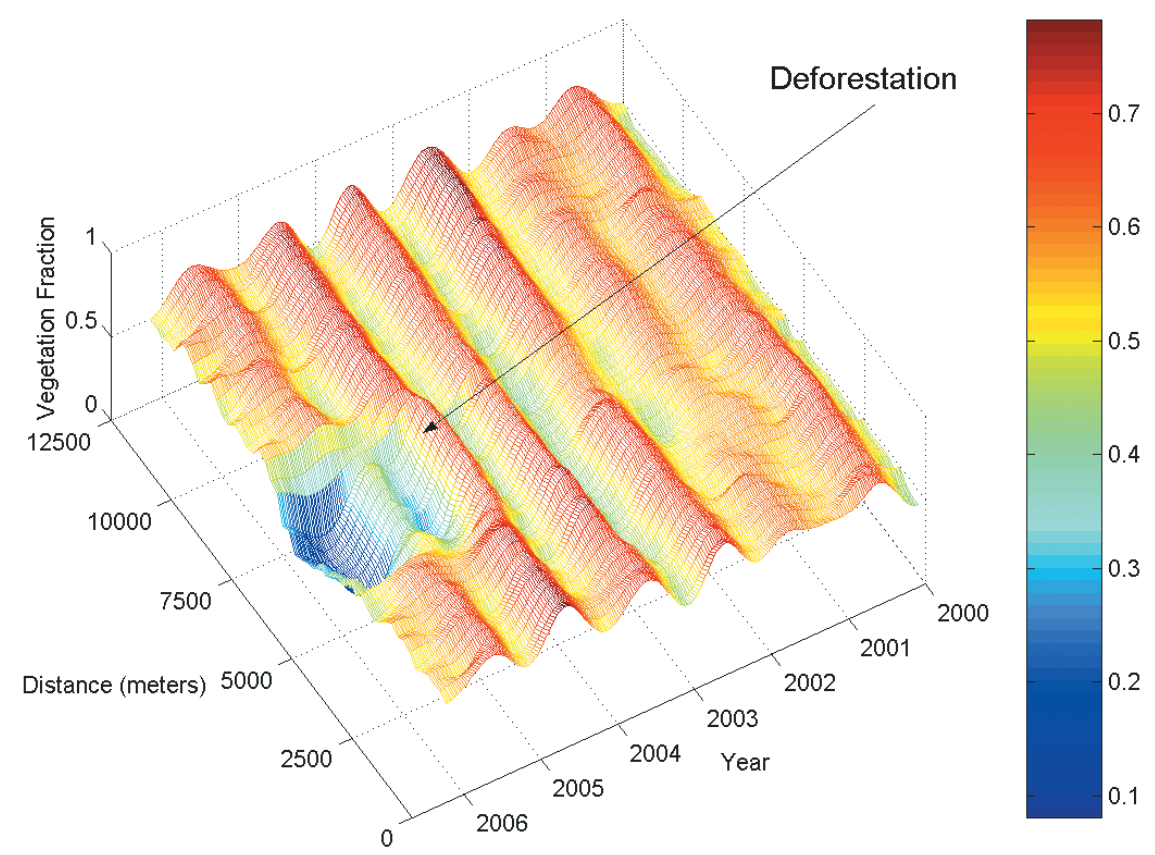

Figure 3 - Transect of deforested area.

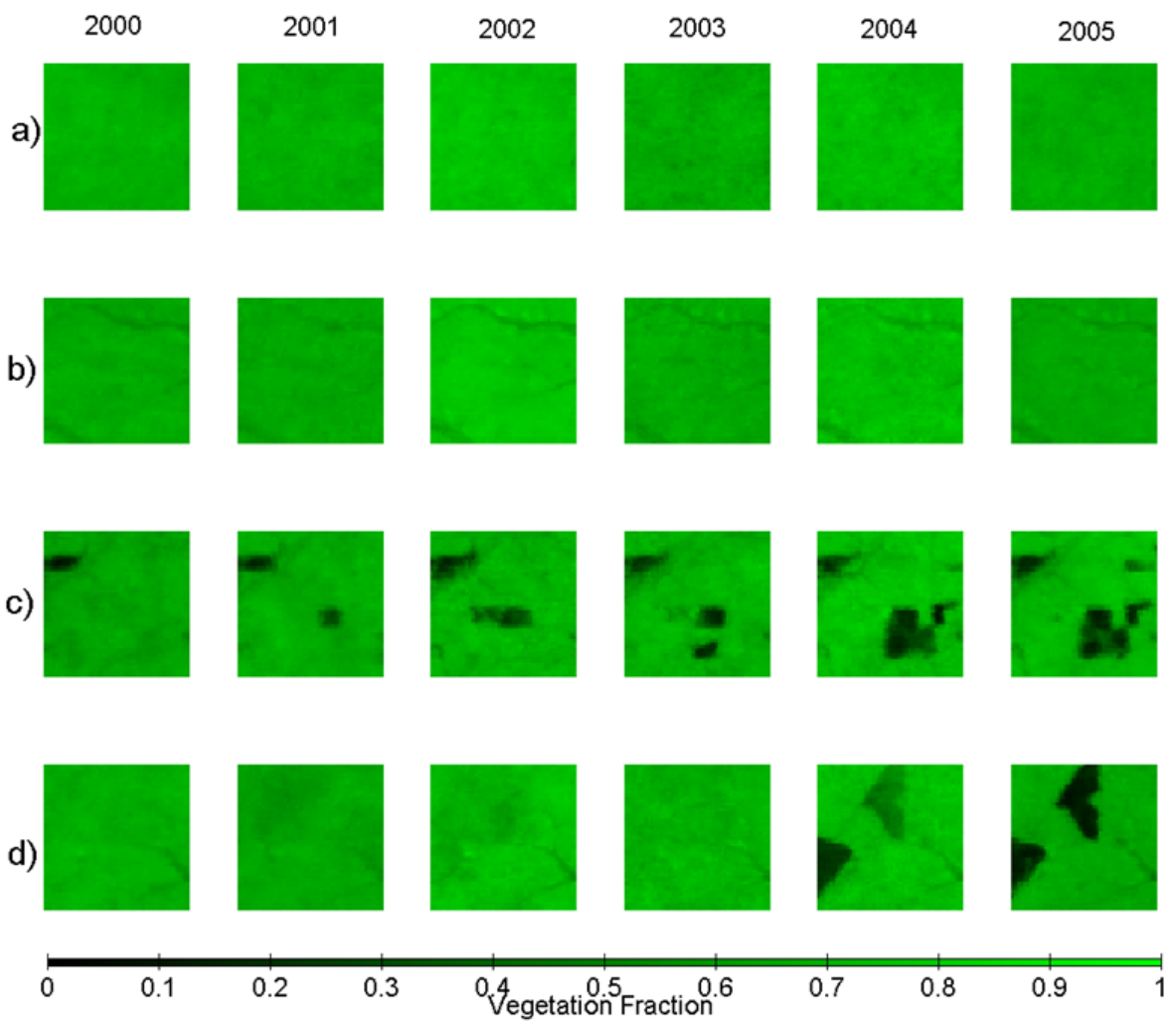

Figure 4 - Examples of vegetation fraction images time-series. Forest area (a) and (b), deforested area (c) and (d). 
precipitation shows temporal lags between wet season and vegetation growing stages. By combining soil and vegetation temporal responses, it can be observed that the post deforestation areas are characterized by high amplitudes of soil fraction that are related to forest biomass reduction.

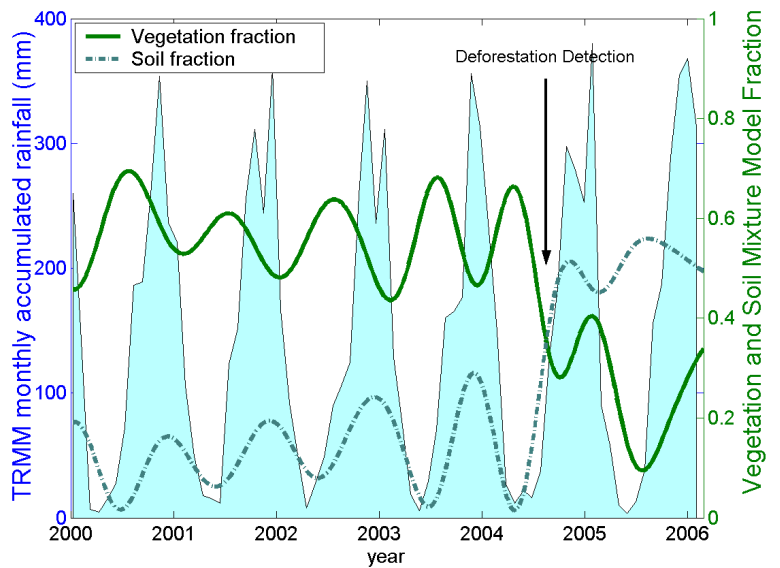

Figure $\mathbf{5}$ - Vegetation fraction cover dynamics of deforestation process.

\section{CONCLUSIONS}

The results of this study indicate that a computational waveletbased method is feasible and practical for MODIS time-series filtering and analysis, specifically for characterizing spatial and temporal patterns in landcover changes. The results show that wavelets transform can provide a gain in multitemporal analysis and visualization on inter-annual fraction images variability patterns. Future work will concentrate on the development of procedures for selecting appropriate thresholds for MODIS time-series filtering.

\section{ACKNOWLEDGMENTS}

This study is part of the Large-Scale Biosphere Atmosphere Experiment in Amazonia (LBA) and was partially supported by the National Institute for Space Research (INPE) and Brazilian National Council of Technological and Scientific Development (CNPq) grant numbers 132140/2005-5 and 307931/2006-4.

\section{REFERENCES}

[1] ABRY P. 1997. Ondelettes et turbulence. Diderot ed., Paris, p. 268.
[2] AGUIAR APD, SHIMABUKURO YE \& MASCARENHAS NDA. 1999. Use of synthetic bands derived from mixing models in the multispectral classification of remote sensing images. International Journal of Remote Sensing, 20: 647-657.

[3] CARREIRAS JMB, SHIMABUKURO YE \& PEREIRA JMC. 2002. FraCtion images derived from SPOT-4 VEGETATION data to assess land-cover change over the State of Mato Grosso, Brazil. International Journal of Remote Sensing, 23: 4979-4983.

[4] CARVALHO LMT. 2001. Mapping and monitoring forest remnants: a multi-scale analysis of spatio-temporal data. $140 \mathrm{p}$. Thesis Wageningen University, Wageningen.

[5] DEFRIES RS, HANSEN MC \& TOWNSHEND JRG. 2000. Global continuous fields of vegetation characteristics: a linear mixture model applied to multi-year $8 \mathrm{~km}$ AVHRR data. International Journal of Remote Sensing, 21: $1389-1414$.

[6] DEFRIES RS, HOUGHTON RA, HANSEN MC, FIELD CB \& SKOLE D. 2002. Carbon emissions from tropical deforestation and regrowth based on satellite observations for the 1980s and 1990s. Proceedings of the National Academy of Sciences of the United States of America, 99(22): 14256-14261, 0ct. 2002.

[7] LI Z \& KAFATOS M. 2000. Interannual Variability of Vegetation in the United States and its Relation to El Niño/Southern Oscillation. Remote Sensing of Environment, 71(3): 239-247.

[8] MEYER Y. 1992. Wavelets and operators. Cambridge Studies in Advanced Math., Cambridge Univ. Press, Cambridge, 37: 223 p.

[9] NATIONAL AERONAUTICS AND SPACE ADMINISTRATION (NASA). Monthly TRMM and Others Data Sources Rainfall Estimate. http://disc2.nascom.nasa.gov/Giovanni/tovas/TRMM_V6.3B43.shtml.

[10] SAKAMOTO T, YOKOZAWA M, TORITANI H, SHIBAYAMA M, ISHITSUKA N \& OHNO H. 2005. A crop phenology detection method using time-series MODIS data. Remote Sensing of Environment, 96(3-4): 366-374.

[11] SHIMABUKURO YE \& SMITH JA. 1991. The least-squares mixing models to generate fraction images derived from remote sensing multispectral data. IEEE Trans. on Geosc. and Rem. Sens., pp. 16-20.

[12] SHIMABUKURO YE, BATISTA GT, MELLO EMK, MOREIRA JC \& DUARTE V. 1998. Using shade fraction image segmentation to evaluate deforestation in Landsat Thematic Mapper images of the Amazon region. International Journal of Remote Sensing, 19(3): 535-541. 